\title{
Quantum statistics and noncommutative black holes
}

$\mathrm{SINP} / \mathrm{TNP} / \mathbf{2 0 1 1 / 0 7}$

\author{
Kumar S. Gupta *, ${ }^{1}$ S. Meljanac ${ }^{\dagger}, 2$ and A. Samsarov ${ }^{\ddagger 2}$ \\ ${ }^{1}$ Theory Division, Saha Institute of Nuclear Physics, 1/AF Bidhannagar, Calcutta 700064, India \\ ${ }^{2}$ Rudjer Bošković Institute, Bijenička c.54, HR-10002 Zagreb, Croatia
}

\begin{abstract}
We study the behaviour of a scalar field coupled to a noncommutative black hole which is described by a $\kappa$-cylinder Hopf algebra. We introduce a new class of realizations of this algebra which has a smooth limit as the deformation parameter vanishes. The twisted flip operator is independent of the choice of realization within this class. We demonstrate that the $R$-matrix is quasi-triangular up to the first order in the deformation parameter. Our results indicate how a scalar field might behave in the vicinity of a black hole at the Planck scale.
\end{abstract}

Keywords: $\kappa$ deformed space, noncommutative black holes, twisted statistics

PACS numbers: 11.10.Nx, 11.30.Cp

\section{INTRODUCTION}

Noncommutative geometry offers a framework for describing the quantum structure of space-time at the Planck scale [1]. Einstein's theory of general relativity together with the uncertainty principle of quantum mechanics leads to a class of models with space-time noncommutativity [2, 3]. The smooth space-time geometry of classical general relativity is thus replaced with a Hopf algebra at the Planck scale. There are many examples of such Hopf algebras including the Moyal plane, $\kappa$-space and Snyder space. The analysis of [2, 3] does not suggest any preferred choice among these models.

Further insight about the possible features of the space-time algebra at the Planck scale comes from the analysis of noncommutative black holes. The algebraic structure associated with a noncommutative black hole can be revealed by studying a simple toy model, such as the noncommutative deformation of the BTZ black hole [4, 5]. The resulting space-time algebra resembles a noncommutative cylinder [6, 7], belonging to the general class of $\kappa$-deformed space-time [8-11]. The appearance of the $\kappa$-cylinder algebra is not restricted to the deformation of the BTZ black hole alone. Such an algebra describes noncommutative Kerr black holes 12] within the framework of twisted gravity theories 13 15]. It also appears in the context of noncommutative FRW cosmologies [16]. In addition, the $\kappa$-Minkowski algebra is relevant in models of doubly-special relativity and in the analysis of astrophysical data from the GRB's [17 30]. This wide-ranging appearance of the $\kappa$-cylinder algebra

\footnotetext{
*kumars.gupta@saha.ac.in

†meljanac@irb.hr

‡asamsarov@irb.hr
}

suggests that it captures certain generic features of noncommutative gravity and black holes and is therefore an interesting toy model to explore Planck scale physics.

In this Letter we shall investigate certain features of the $\kappa$-cylinder algebra using a scalar field as a simple probe. In order to study quantum field theory in any space-time, it is essential to specify the statistics of the quantum field. It has been known for a long time that quantum gravity can admit exotic statistics [31 33]. More recently, the idea of twisted statistics and the associated $R$-matrices have appeared in the context of quantum field theories in noncommutative space-time [34 39], including the $\kappa$-deformed spaces [40 49]. To this end, it is useful to work with realizations of the $\kappa$-space and the associated star products [51 55]. In this letter we introduce a new class of realizations of the $\kappa$-cylinder algebra which has a smooth limit as the deformation parameter vanishes, which is different from the previous discussion in the literature [6, 7]. In addition, we obtain the twisted flip operator and show that it is independent of the particular choice of realization within the class of realizations considered here.

For the commutative theories of gravity, probing a black hole space-time with a scalar field yields rich information about the underlying geometry [56 58$]$. In our context, this amounts to studying a scalar field coupled to the $\kappa$-cylinder algebra [42, 45 47, 49, 50]. Here we analyze this problem for a class of realizations of the $\kappa$ cylinder algebra that has a smooth limit as the deformation parameter vanishes. We find that the creation and annihilation operators appearing in the mode expansion of the scalar field satisfy a twisted oscillator algebra. This provides an initial glimpse as to how a scalar field might behave in the vicinity of a black hole at the Planck scale.

This Letter is organized as follows. In Section 2 we review certain features of the noncommutative black holes that are relevant for our work. In Section 3 we discuss 
the realizations of the $\kappa$-Minkowski algebra and introduce a new class of realizations for the $\kappa$-cylinder algebra that has a smooth limit as the deformation parameter vanishes. We also discuss the star products and the twists associated with this algebra. In Section 4 we discuss the twisted statistics and the $R$-matrices for the realizations discussed here. The twisted oscillator algebra for the class of realizations considered in this Letter is derived in Section 5. In Section 6 we conclude the paper with some discussions and an outlook.

\section{NONCOMMUTATIVE BLACK HOLES}

We start by reviewing certain features of noncommutative BTZ black hole [4, 5] which are useful for our analysis. In the commutative case, the metric for a nonextremal BTZ black hole in terms of the Schwarzschild like coordinates $(r, \phi, t)$ is given by $[59,60]$

$$
\begin{aligned}
d s^{2} & =\left(M-\frac{r^{2}}{\ell^{2}}-\frac{J^{2}}{4 r^{2}}\right) d t^{2}+\left(-M+\frac{r^{2}}{\ell^{2}}+\frac{J^{2}}{4 r^{2}}\right)^{-1} d r^{2} \\
& +r^{2}\left(d \phi-\frac{J}{2 r^{2}} d t\right)^{2}
\end{aligned}
$$

where $0 \leq r<\infty,-\infty<t<\infty, 0 \leq \phi<2 \pi$, and $M$ and $J$ are respectively the mass and spin of the black hole and $\Lambda=-1 / \ell^{2}$ is the cosmological constant. Here we restrict our attention to the non-extremal case, where the two horizons $r_{ \pm}$are given by

$$
r_{ \pm}^{2}=\frac{M \ell^{2}}{2}\left\{1 \pm\left[1-\left(\frac{J}{M \ell}\right)^{2}\right]^{\frac{1}{2}}\right\}
$$

The BTZ black hole can also be obtained by quotienting the manifold $A d s_{3}$ or $S L(2, \mathbb{R})$ by a discrete subgroup of its isometry. A noncommutative version of the BTZ black hole can be realized by a quantum deformation of $A d s_{3}$ or $S L(2, \mathbb{R})$ in such a way that is compatible with the quotienting [4, 5]. In the resulting noncommutative theory, the coordinates $r, \phi$ and $t$ are replaced by the corresponding operators $\hat{r}, \hat{\phi}$ and $\hat{t}$ respectively, which no longer commute but satisfy the algebra

$$
\left[e^{i \hat{\phi}}, \hat{t}\right]=\theta e^{i \hat{\phi}} \quad[\hat{r}, \hat{t}]=\left[\hat{r}, e^{i \hat{\phi}}\right]=0,
$$

where the constant $\theta$ is proportional to $\ell^{3} /\left(r_{+}^{2}-r_{-}^{2}\right)$. The algebra (3) is so constructed that its cetral elements are kept invariant under the action of the isometry group of the BTZ black hole [4]. We therefore see that the noncommutative BTZ black hole is equivalent to a noncommutative cylinder.

From (3) we find that the operator $\hat{r}$ is in the centre of the algebra. What is perhaps not so obvious is that the operator $e^{-2 \pi i \hat{t} / \theta}$ also belongs to the centre of this algebra. As a result, in any irreducible representation of (3), the element $e^{-2 \pi i \hat{t} / \theta}$ is proportional to the identity,

$$
e^{-2 \pi i \hat{t} / \theta}=e^{i \sigma} 1
$$

where the constant parameter $\sigma \in \mathbb{R} \bmod (2 \pi)$. Eqn. (4) implies that in any irreducible representation of (3), the spectrum of the $\hat{t}$ operator is quantized [6, 7, 61] and is given by

$$
\text { spec } \hat{t}=n \theta-\frac{\sigma \theta}{2 \pi}, \quad n \in \mathbb{Z} .
$$

As mentioned before, a similar algebra as in (3) appears in the noncommutative generalization of the rotating Kerr black hole [12] as well as for the noncommutative version of the FRW cosmology [16]. For the rest of this paper we shall consider the algebra in (3) as a prototype for noncommutative black holes and study its properties.

\section{REALIZATIONS OF $\kappa$-MINKOWSKI SPACE}

\subsection{Generalities}

We start by recalling certain general properties of the $\kappa$-Minkowski algebra relevant for our analysis. The operators corresponding to coordinates on $\kappa$-deformed noncommutative space satisfy the relation

$$
\left[\hat{x}_{\mu}, \hat{x}_{\nu}\right]=i\left(a_{\mu} \hat{x}_{\nu}-a_{\nu} \hat{x}_{\mu}\right),
$$

where $a_{0}, a_{1}, a_{2}, \ldots, a_{n-1}$ appearing in (6) are real constant parameters describing a deformation of Minkowski space. It is understood that the Greek indices run through the set $\{0,1, \ldots, n-1\}$, and the latin indices run through the subset $\{1,2, \ldots, n-1\}$ with the summation over repeating indices assumed.

Next we introduce derivative operators with the properties

$$
\begin{aligned}
& {\left[\partial_{\mu}, \partial_{\nu}\right]=0,} \\
& {\left[\partial_{\mu}, \hat{x}_{\nu}\right]=\phi_{\mu \nu}(\partial),}
\end{aligned}
$$

where the functions $\phi_{\mu \nu}(\partial)$ in derivatives here provide realizations of noncommutative coordinates in the auxiliary Hilbert space $\mathcal{H}\left(\mathbb{R}^{1,3}, d^{4} x\right)$, which is spanned by the ordinary commutative coordinates

$$
\left[x_{\mu}, x_{\nu}\right]=0, \quad\left[\partial_{\mu}, x_{\nu}\right]=\delta_{\mu \nu} .
$$

We also define a shift operator which satisfy

$$
\left[Z, \hat{x}_{\mu}\right]=i a_{\mu} Z, \quad\left[Z, \partial_{\mu}\right]=0 .
$$

The realizations of noncommutative coordinates can then be written in the form [51, 52]

$$
\hat{x}_{\mu}=x^{\alpha} \phi_{\alpha \mu}(\partial) .
$$

We restrict our attention to deformation parameters with zero spatial components, $a_{\mu}=a_{0} \delta_{0 \mu}, a_{0} \neq 0$. Then the commutation relations become

$$
\left[\hat{x}_{i}, \hat{x}_{j}\right]=0, \quad\left[\hat{x}_{0}, \hat{x}_{j}\right]=i a_{0} \hat{x}_{j},
$$


The algebra (12) admits the realization of the type (11), which also includes the class of noncovariant realizations given by [62]

$$
\begin{aligned}
& \hat{x}_{0}=x_{0} \psi(A)+i a_{0} x_{k} \partial_{k} \gamma(A), \\
& \hat{x}_{i}=x_{i} \varphi(A), \quad \gamma=\frac{\varphi^{\prime}}{\varphi} \psi+1,
\end{aligned}
$$

where $A=-i a_{0} \partial_{0}$. The above realization is parametrized by two real-analytic functions $\varphi$ and $\psi$ satisfying the boundary conditions $\varphi(0)=\psi(0)=1$ and $\varphi^{\prime}(0)$ is finite. The shift operator has the form

$$
Z=e^{\Psi(A)}, \quad \Psi(A)=\int_{0}^{A} \frac{d t}{\psi(t)} .
$$

The derivatives $\partial_{\mu}$ generate the action on $\kappa$-space coordinates, which, together with angular momenta form a deformed Poincaré algebra that has a Hopf algebra structure. The deformed coproducts are given by

$$
\Delta A=\Psi^{-1} \circ \ln (Z \otimes Z) .
$$

and

$\Delta \partial_{0}=\frac{i}{a_{0}} \Delta A, \quad \Delta \partial_{i}=\varphi(\Delta A)\left(\frac{\partial_{i}}{\varphi(A)} \otimes 1+Z \otimes \frac{\partial_{i}}{\varphi(A)}\right)$,

$$
\Delta Z=Z \otimes Z
$$

We note that the coproduct of $M_{i j}$ is undeformed.

\subsection{Smooth realizations of the $\kappa$-cylinder algebra}

We now focus on the algebra relevant for the noncommutative black hole introduced in Section 2. Our aim is to investigate a version of $\kappa$-deformed noncommutative space, where the only space coordinate is compactified to a circle, a type of manifold known as noncommutative cylinder. The noncommutative cylinder is generally specified by the three noncommutative coordinates, $\hat{t}, \hat{z}$ and $\hat{z}^{\dagger}$, which can be considered as noncommutative versions of the real parameter $t$ and two complex parameters $z=\rho e^{i \phi}$ and its complex conjugate $\bar{z}=\rho e^{-i \phi}$, respectively $^{1}$.

$$
\left[\hat{z}, \hat{z}^{\dagger}\right]=0, \quad[\hat{t}, \hat{z}]=a_{0} \hat{z}, \quad\left[\hat{t}, \hat{z}^{\dagger}\right]=-a_{0} \hat{z}^{\dagger} .
$$

These operators satisfy the same constraint equation as in the commutative case, namely $\hat{z} \hat{z}^{\dagger}=\rho^{2}$. It is easy to see that the algebra (18) can be recovered from (6) by

\footnotetext{
${ }^{1}$ In the rest of the paper we assume the notation $x_{\alpha}=\left(x_{0}=\right.$ $\left.t, x_{1}=z\right)$ and $\hat{x}_{\alpha}=\left(\hat{x}_{0}=\hat{t}, \hat{x}_{1}=\hat{z}\right)$, keeping track with the notation in terms of cylinder coordinates.
}

restricting description effectively to only two noncommutative coordinates, $\hat{x}_{0} \equiv \hat{t}, \quad \hat{x}_{1} \equiv \hat{z}$ and by letting deformation parameter $a_{0}$ to change into $-i a_{0}$. This simple prescription accommodates for the compactification of the only space coordinate present in the model. This algebra (18) admits a class of realizations which has a smooth limit, which is given by

$$
\begin{aligned}
\hat{z} & =\rho e^{i \phi} e^{i h(A)}, \\
\hat{z}^{\dagger} & =\rho e^{-i \phi} e^{-i h(A)}, \\
\hat{t} & =t \psi(A)-i a_{0} \partial_{\phi} \gamma(A),
\end{aligned}
$$

with functions $\psi, h$ satisfying the boundary conditions $h(0)=0$ and $\psi(0)=1$, respectively. Additionally, $h(A)$ is understood to be a hermitian operator $h(A)^{\dagger}=h(A)$. Consistency requires that

$$
\gamma(A)=\psi(A) \frac{d h}{d A}+1 .
$$

The shift operator $Z$ is then a unitary operator, $Z^{-1}=$ $Z^{\dagger}$, which is given by

$$
Z=e^{+i \int \frac{d t}{\psi(t)}}
$$

and has the properties

$$
[Z, \hat{t}]=a_{0} Z, \quad[Z, \hat{z}]=\left[Z, \hat{z}^{\dagger}\right]=0 .
$$

Note that in Eq. (19), instead of exponential operator $e^{i h(A)}$, one could generally choose some function $\varphi(A)$, such that $\varphi^{\dagger}(A) \varphi(A) \geq 0$, to comply with the general form (14). In this case one would have $\hat{z} \hat{z}^{\dagger}=$ $\rho^{2} \varphi^{\dagger}(A) \varphi(A)$. There are two particularly interesting situations where the results, together with the implication on statistics, can be given in full detail. These situations include choices $i) \psi(A)=1$ and $i i) \gamma(A)=\gamma_{0}, \gamma_{0}$ being some constant. The first choice is elaborated for $\kappa$-Minkowski space in [48],[51],[53] and for the second one an appropriate analysis has been done in [64].

\subsection{Star product}

For each $\varphi$-realization and corresponding ordering of the $\kappa$-Minkowski algebra, there exists a unique star product $\star_{\varphi}$, twist operator $\mathcal{F}_{\varphi}$ and a coproduct $\Delta_{\varphi}$. The star product in $\varphi$ realization between two functions $f$ and $g$ in the algebra of functions on $R^{n}$, is generally given by

$$
f \star_{\varphi} g=m_{0}\left(\mathcal{F}_{\varphi} f \otimes g\right),
$$

where $m_{0}$ is the multiplication map in the Hopf algebra, namely, $m_{0}(f \otimes g)=f g$ and $\mathcal{F}_{\varphi}$ is the twist element. This can also be written in the form [51], 53]

$$
\left(f \star_{\varphi} g\right)(x)=\lim _{u \rightarrow x} m_{0}\left(e^{x^{\alpha}\left(\triangle_{\varphi}-\triangle_{0}\right) \partial_{\alpha}} f(u) \otimes g(u)\right),
$$


where the coproduct $\triangle_{\varphi}$ for translation generators is given in (17) and

$$
\triangle_{0}(\partial)=\partial \otimes 1+1 \otimes \partial
$$

is the untwisted coproduct. The coproduct $\triangle_{\varphi}$ can also be obtained by twisting the primitive coproduct $\triangle_{0}$,

$$
\triangle_{\varphi}=\mathcal{F}_{\varphi}^{-1} \triangle_{0} \mathcal{F}_{\varphi}
$$

where the twist element $\mathcal{F}_{\varphi}$ follows from rearranging the operator $e^{x^{\alpha}\left(\Delta_{\varphi}-\Delta_{0}\right) \partial_{\alpha}}$ with the help of certain mathematical identity [48], 53].

We shall now restrict to the case $i) \psi(A)=1$. The coproduct in this case can be obtained from Eq. (17) by specializing it to $\psi(A)=1$. This coproduct then directly determines the star product in $\kappa$-space, according to relation (26), which finally allows us to identify the corresponding twist operator as

$$
\mathcal{F}_{\varphi}=e^{(N \otimes 1) \ln \frac{\varphi(A \otimes 1+1 \otimes A)}{\varphi(A \otimes 1)}+(1 \otimes N)\left(A \otimes 1+\ln \frac{\varphi(A \otimes 1+1 \otimes A)}{\varphi(1 \otimes A)}\right)}
$$

where $N=x_{i} \partial / \partial x_{i} \equiv x_{i} \partial_{i}$ is the dilatation generator with summation going over space indices only and $A=$ $-a_{0} \partial_{0}$. When written in terms of cylinder coordinates, these operators look as $N=z \partial_{z}=-i \partial_{\phi}$ and $A=$ $-a_{0} \partial_{t}$, respectively.

We point out that the star product and the twist element depend explicitly on the choice of the ordering. For $\phi(A)=e^{-c A}$ where $c \in \mathbb{R}$, we obtain a simple interpolation between right ordering $(c=0)$ and left ordering $(c=1)$, with the twist operator given by

$$
\mathcal{F}_{c}=e^{-c N \otimes A+(1-c) A \otimes N} .
$$

For $c=\frac{1}{2}$, we have a symmetric ordering, which is completely different from the totally symmetric Weyl ordering 63]. Using $\triangle N=N \otimes 1+1 \otimes N, \triangle A=A \otimes 1+1 \otimes A$, and $[N, A]=0$, it is easy to verify that the above class of twist operators $\mathcal{F}_{c}$ satisfies the cocycle condition

$$
\left(\mathcal{F}_{c} \otimes 1\right)(\triangle \otimes 1) \mathcal{F}_{c}=\left(1 \otimes \mathcal{F}_{c}\right)(1 \otimes \triangle) \mathcal{F}_{c}
$$

for all $c \in \mathbb{R}$.

\section{NONCOMMUTATIVE BLACK HOLES AND PARTICLE STATISTICS}

Quantum gravity admits the possibility of unusual particle statistics, with associated implications for the spin-statistics connection [31 33]. Within the paradigm of noncommutative geometry as a possible description of space-time at the Planck scale [2, 3], and the $\kappa$ Minkowski algebra as a possible description of a class of noncommutative black holes [4, 5, 12, 16], it is interesting to ask how the particle statistics would be described in this framework.
Let us first consider the commutative flip operator $\tau_{0}$ associated with the exchange of particles, given by

$$
\tau_{0}(f \otimes g)=g \otimes f,
$$

which satisfies the idempotency property, $\tau_{0}^{2}=1 \otimes 1$. In the commutative case, the statistics is superselected. This is expressed by the condition that coproduct $\Delta_{0}(\Lambda)$ of any generator $\Lambda$ of the Poincare algebra commutes with the flip operator $\tau_{0}$,

$$
\left[\Delta_{0}(\Lambda), \tau_{0}\right]=0
$$

In the noncommutative case, the coproduct of $\Lambda$ is given by

$$
\Delta_{\varphi}(\Lambda)=\mathcal{F}_{\varphi}^{-1} \Delta_{0}(\Lambda) \mathcal{F}_{\varphi}
$$

However, the commutative flip operator $\tau_{0}$ does not commute with $\Delta_{\varphi}(\Lambda)$. If we assume that particle statistics continues to be superselected in the noncommutative case, then we must find a new twisted flip operator which will commute with $\Delta_{\varphi}(\Lambda)$. Such a twisted flip operator can be defined and is given by

$$
\tau_{\varphi}=\mathcal{F}_{\varphi}^{-1} \tau_{0} \mathcal{F}_{\varphi}
$$

so that the action of deformed symmetry group respects new statistics

$$
\left[\Delta_{\varphi}(\Lambda), \tau_{\varphi}\right]=0
$$

Having defined the twisted flip operator, the symmetrization and antisymmetrization can now be carried out with the projection operators $\frac{1}{2}\left(1 \pm \tau_{\varphi}\right)$, respectively. This would ensure that the twisted statistics of a two particle state in the $\kappa$ space would remain unchanged under the action of the twisted symmetry group.

For this particular special class of $\varphi$-realizations, the twist element is given in (29). Using (29) and (35), we obtain an explicit expression for the twisted flip operator $\tau_{\varphi}$ for the class of realizations $\psi(A)=1$ as

$$
\tau_{\varphi}=e^{N \otimes A-A \otimes N} \tau_{0} .
$$

The last relation makes possible to identify the $R$-matrix and the corresponding $r$-matrix that is given by

$$
r=\frac{i}{a_{0}}(N \otimes A-A \otimes N)
$$

One can check that this $r$-matrix satisfies the classical Yang-Baxter equation.

\section{TWISTED OSCILLATOR ALGEBRA IN THE NONCOMMUTATIVE BLACK HOLE BACKGROUND}

In the commutative theory, probing a black hole geometry with a scalar field provides important information 
about the space-time structure. Here we initiate a similar study for the noncommutative black hole. In order to study the behaviour of a quantum field around a noncommutative black hole, it is essential to know the algebra of the creation and annihilation operators. Below we obtain the oscillator algebra in the background of a noncommutative black hole, for the particular class of realizations discussed in this Letter.

As a starting point we take the relation

$$
f \otimes g=\tau_{\varphi}(f \otimes g)
$$

which is consistent with the idempotency property of the statistics flip operator, $\tau_{\varphi}^{2}=1 \otimes 1$. The statistics flip operator (37) in conjuction with Eqn.(39) leads to the following condition:

$$
\phi(x) \otimes \phi(y)-e^{-(A \otimes N-N \otimes A)} \phi(y) \otimes \phi(x)=0 .
$$

It is obvious that condition (40) is independent of the realization.

In order to acquire further insight of the physics of $\kappa$ cylinder, we stick to the particular choice $\varphi=e^{-\frac{A}{2}}=$ $e^{-\frac{a_{0} \partial_{0}}{2}}$, i.e. $h(A)=-\frac{A}{2 i}$. This particular realization corresponds to symmetric ordering of coordinates and it gives rise to a generalized Klein-Gordon equation [49],

$$
\left[\partial_{z} \partial_{\bar{z}}-\frac{4}{a_{0}^{2}} \sinh ^{2}\left(\frac{a_{0} \partial_{0}}{2}\right)-m^{2}\right] \Phi=0
$$

describing a matter field $\Phi(x)$ which probes a black hole background. The field $\Phi(x)$ can be decomposed in positive and negative frequency modes as

$$
\Phi(x)=\int \frac{d p_{z}}{\sqrt{p_{z}^{2}+m^{2}}}\left[b\left(\omega, p_{z}\right) e^{-i p \cdot x}+b^{\dagger}\left(\omega, p_{z}\right) e^{i p \cdot x}\right]
$$

where $b^{\dagger}\left( \pm \omega, p_{z}\right)=b^{\dagger}\left(\mp \omega, p_{z}\right)$. Here we have anticipated that momentum $p$ is a two-vector $p=\left(p_{0}, p_{z}\right)$, with two components $p_{0}$ and $p_{z}$ being canonically conjugated to cylinder coordinates $t$ and $z$, respectively. The generalized Klein-Gordon equation (41) fixes the form of the dispersion relation

$$
p_{0}^{ \pm}= \pm \omega= \pm \frac{2 i}{a_{0}} \sinh ^{-1}\left(\frac{a_{0}}{2 i} \sqrt{p_{z}^{2}+m^{2}}\right) .
$$

This expression encodes the on-shell condition for the particles and determines the energies of the positive and negative frequency modes in the Fourier expansion (42). In a case we have a product of two bosonic fields $\phi(x)$ and $\phi(y)$, both having mode expansion (42), a straightforward application of the Eqn.(40) leads to a deformed oscillator algebra

$$
\begin{array}{r}
b^{\dagger}\left(p_{0}, p_{z}\right) b\left(q_{0}, q_{z}\right)-e^{i a_{0}\left(q_{0} \partial_{p_{z}} p_{z}+\partial_{q z} q_{z} p_{0}\right)} b\left(q_{0}, q_{z}\right) b^{\dagger}\left(p_{0}, p_{z}\right)=-\delta^{(2)}(p-q) \\
b^{\dagger}\left(p_{0}, p_{z}\right) b^{\dagger}\left(q_{0}, q_{z}\right)-e^{i a_{0}\left(-q_{0} \partial_{p_{z}} p_{z}+\partial_{q_{z}} q_{z} p_{0}\right)} b^{\dagger}\left(q_{0}, q_{z}\right) b^{\dagger}\left(p_{0}, p_{z}\right)=0 \\
b\left(p_{0}, p_{z}\right) b\left(q_{0}, q_{z}\right)-e^{i a_{0}\left(q_{0} \partial_{p_{z}} p_{z}-\partial_{q_{z}} q_{z} p_{0}\right)} b\left(q_{0}, q_{z}\right) b\left(p_{0}, p_{z}\right)=0 .
\end{array}
$$

where the time components of the momenta, $p_{0}$ and $q_{0}$, are given in Eqn. (43).

The creation and annihilation operators satisfying the above given deformed commutation relations are the ones appearing in the mode decomposition of the scalar field satisfying the generalized Klein-Gordon Eqn.(41). This generalized Klein-Gordon equation is invariant under the $\kappa$-Poincaré algebra.

Since deformed statistics flip operator is idempotent, we know that the statistics is governed by the permutation group and not by the more general braided group. In this case the equation (39) identifies the eigenspace of $\tau_{\varphi}$ corresponding to eigenvalue +1 , that is, it describes deformed bosons, i.e. particles that obey commutation relations (44,46). On the other hand, the projector $\frac{1}{2}\left(1-\tau_{\varphi}\right)$ determines the eigenspace of $\tau_{\varphi}$ with eigenvalue -1 , thus defining particles with deformed Fermi-Dirac statistics. As for condition (39), which was shown to lead to the set of commutatation relations (44,46) describing defor- med bosons, the (anti)commutatation relations obeyed by deformed fermions can be deduced from the condition

$$
f \otimes g=-\tau_{\varphi}(f \otimes g)
$$

The later condition leads to the same type of relations as (44,46), except only for - sign being replaced by the + sign. It gives at least a part of the eigenspace for $\tau_{\varphi}$ with eigenvalue -1 , thus describing deformed fermions.

The dispersion relation

$$
p_{z}^{2}+m^{2}=\frac{4}{a_{0}^{2}} \sinh ^{2}\left(\frac{a_{0} p_{0}}{2 i}\right) \equiv \epsilon^{2}
$$

is readily obtained from (43). It is valid for both, deformed bosons and fermions. And even more, with the help of it we can deduce the form of equation governing dynamics of deformed fermions by linearizing [65] the Klein-Gordon equation (41). Indeed, the dispersion (48) has the form of standard energy-momentum relation once 
we make identification $\epsilon=\frac{2}{a_{0}} \sinh \left(\frac{a_{0} p_{0}}{2 i}\right)$. This enables us to write down the Dirac equation in momentum space as

$\epsilon u\left(p_{0}, p_{z}\right) \equiv \frac{2}{a_{0}} \sinh \left(\frac{a_{0} p_{0}}{2 i}\right) u\left(p_{0}, p_{z}\right)=(\vec{\alpha} \cdot \vec{p}+\beta m) u\left(p_{0}, p_{z}\right)$

where $\alpha^{i}=-\gamma^{0} \gamma^{i}, \beta=\gamma^{0}$ are Dirac $\gamma$ matrices and $u\left(p_{0}, p_{z}\right)$ is a two-component spinor. Note that in our $1+1$-dim case $\vec{\alpha} \cdot \vec{p}=\alpha^{1} p_{z}$ and $\alpha^{i}$ and $\beta$ reduce to Pauli matrices and $2 \times 2$ identity matrix, respectively. The equation (49) is not linear any more. It is nonlinear and its coordinate space counterpart is non-local and thus hard to analyse. The nonlocal operator can however be expanded in terms of deformation parameter $a_{0}$ and correspondingly obtained equation can be analysed within the same order. This analysis is out of scope of the current paper and is planned for future publication. Nevertheless, we see that dynamics of particles in the background of the noncommutative BTZ black hole is described in terms of equations that are nonlocal. This happened to be the case for deformed bosons (see Eq.(411) as well as for deformed fermions (see Eq.(49)). The extraction of any further information concerning the particle dynamics will thus innevitably require perturbation calculus.

The situation described also means that other objects in our approach, such as the twist, the $R$-matrix and the statistics flip operator will eventually have to be deduced through the perturbation procedure as well. To illustrate how this can be done, we investigate a quasi-triangular structure of the symmetry algebra in the particular noncommutative background described by

$$
\begin{aligned}
& \hat{x}_{i}=x_{i}(1+i \alpha(a \partial))+i \beta(a x) \partial_{i}, \\
& \hat{x}_{0}=x_{0}(1+i \delta(a \partial))+i a_{0} \gamma(x \partial) .
\end{aligned}
$$

The above realizations are written perturbatively up to first order in deformation $a$ and already include the previ- ously analyzed realizations (13) and (14) up to the same order. Indeed, the later is obtained from the former for $\beta=\gamma+\delta=0$. The quasi-triangular structure is of importance since it gives rise to statistical properties of particles in this noncommutative background. To obtain it, we have to find the twist operator, which can be constructed once the coproducts for derivatives are known. The equations (50), (51) lead to coproducts

$$
\Delta\left(\partial_{i}\right)=\partial_{i} \otimes 1+1 \otimes \partial_{i}-i \gamma a_{0} \partial_{0} \otimes \partial_{i}-i \alpha a_{0} \partial_{i} \otimes \partial_{0}+\mathcal{O}\left(a^{2}\right) .
$$

$$
\Delta\left(\partial_{0}\right)=\partial_{0} \otimes 1+1 \otimes \partial_{0}-i(\gamma+\delta) a_{0} \partial_{0} \otimes \partial_{0}+i \beta a_{0} \partial_{i} \otimes \partial_{i}+\mathcal{O}\left(a^{2}\right) .
$$

The twist element can be now deduced from the exponential factor in (26). However, since there is an ambiguity as where to place the coordinate variable $x^{\alpha}$ (whether to attach it to the first or to the second factor in the tensor product expansion of the exponential), we write the twist element in a sufficiently general form to accommodate for this indeterminacy,

$$
\begin{aligned}
\mathcal{F} & =1 \otimes 1-\lambda_{1} i \beta a_{0} x_{0} \partial_{i} \otimes \partial_{i}-\left(1-\lambda_{1}\right) i \beta a_{0} \partial_{i} \otimes x_{0} \partial_{i} \\
& +\lambda_{2} i(\gamma+\delta) a_{0} x_{0} \partial_{0} \otimes \partial_{0}+\left(1-\lambda_{2}\right) i(\gamma+\delta) a_{0} \partial_{0} \otimes x_{0} \partial_{0} \\
& -\lambda_{3} i \gamma a_{0} x_{i} \partial_{0} \otimes \partial_{i}-\left(1-\lambda_{3}\right) i \gamma a_{0} \partial_{0} \otimes x_{i} \partial_{i} \\
& -\lambda_{4} i \alpha a_{0} x_{i} \partial_{i} \otimes \partial_{0}-\left(1-\lambda_{4}\right) i \alpha a_{0} \partial_{i} \otimes x_{i} \partial_{0}+\mathcal{O}\left(a^{2}\right)
\end{aligned}
$$

where $\lambda_{1}, \lambda_{2}, \lambda_{3}, \lambda_{4}$, are free parameters, whose presence reflects the above described indeterminacy. They indicate the weight with which coordinate $x^{\alpha}$ is attached to the first, i.e. to the second part of the tensor product in (54). It is now straightforward to show that coproducts (52) and (53) can be reproduced from primitive coproducts (27) via twist (54),

$$
\Delta\left(\partial_{\mu}\right)=\mathcal{F}^{-1} \Delta_{0}\left(\partial_{\mu}\right) \mathcal{F},
$$

for any choice of free parameters $\lambda_{1}, \lambda_{2}, \lambda_{3}, \lambda_{4}$.

The statistics flip operator in this setting can be calculated from (35) by using twist element (54), leading finally to the $R$-matrix in the first order in $a_{0}$, given by

$$
\begin{aligned}
R & =1 \otimes 1+\left(2 \lambda_{1}-1\right) i \beta a_{0}\left(M_{0 i} \otimes \partial_{i}-\partial_{i} \otimes M_{0 i}\right)+\left(\left(2 \lambda_{1}-1\right) \beta+\lambda_{3} \gamma+\lambda_{4} \alpha-\alpha\right) i a_{0}\left(x_{i} \partial_{0} \otimes \partial_{i}-\partial_{i} \otimes x_{i} \partial_{0}\right) \\
& +\left(\lambda_{4} \alpha+\lambda_{3} \gamma-\gamma\right) i a_{0}\left(x_{i} \partial_{i} \otimes \partial_{0}-\partial_{0} \otimes x_{i} \partial_{i}\right)+\left(1-2 \lambda_{2}\right) i(\gamma+\delta) a_{0}\left(x_{0} \partial_{0} \otimes \partial_{0}-\partial_{0} \otimes x_{0} \partial_{0}\right)+\mathcal{O}\left(a^{2}\right)
\end{aligned}
$$

where $M_{0 i}=x_{0} \partial_{i}-x_{i} \partial_{0}$ in the lowest order in $a_{0}$. It satisfies quantum Yang-Baxter equation in first order of deformation $a_{0}$, for every choice of free parameters $\lambda_{1}, \lambda_{2}, \lambda_{3}, \lambda_{4}$. Here we can use the known result (38) to fix, at least partially, the values of the free parame- ters. Thus, by noting that the realization (13), (14), expanded to the first order in $a_{0}$, emerges from the realization (50), (51) under the condition $\beta=\gamma+\delta=0$, it is clear that the quasi-triangular structure (56) reduces to classical $r$-matrix (38) under the same condition, 
$\beta=\gamma+\delta=0$. From this, we can fix the parameters $\lambda_{3}$ and $\lambda_{4}, \lambda_{3}=0, \lambda_{4}=1$, while $\lambda_{1}$ and $\lambda_{2}$ still remain undetermined. The analysis thus shows that for a given realization we get the whole family of $R$-matrices. Are these $R$-matrices mutualy equivalent or at least, is there any relation between them is still an open question that is the subject of our current investigation. We hope to address these issues in a near future.

Applying the above results to the $\kappa$-cylinder case, in the first order of deformation parameter $a_{0}$ we have the following realizations

$$
\begin{gathered}
\hat{z}=z\left(1-\alpha a_{0} \partial_{t}\right)-\beta\left(a_{0} t\right) \partial_{z}, \\
\hat{t} \equiv \hat{x}_{0}=t\left(1-(\gamma+\delta) a_{0} \partial_{t}\right)-i a_{0} \gamma \partial_{\phi},
\end{gathered}
$$

leading to coproducts

$\Delta\left(\partial_{t}\right)=\Delta_{0}\left(\partial_{t}\right)-(\gamma+\delta) a_{0} \partial_{t} \otimes \partial_{t}+\beta a_{0} \partial_{z} \otimes \partial_{z}+\mathcal{O}\left(a^{2}\right)$, $\Delta\left(\partial_{z}\right)=\Delta_{0}\left(\partial_{z}\right)-\gamma a_{0} \partial_{t} \otimes \partial_{z}-\alpha a_{0} \partial_{z} \otimes \partial_{t}+\mathcal{O}\left(a^{2}\right)$.

The variable $\phi$ is a polar angle used to parametrize the cylindrical coordinate $z$. The relevant $R$-matrix now appears to be

$$
\begin{aligned}
R & =1 \otimes 1+\left(2 \lambda_{1}-1\right) \beta a_{0}\left(M_{t z} \otimes \partial_{z}-\partial_{z} \otimes M_{t z}\right) \\
& +\left(2 \lambda_{1}-1\right) \beta a_{0}\left(z \partial_{t} \otimes \partial_{z}-\partial_{z} \otimes z \partial_{t}\right) \\
& -i(\alpha-\gamma) a_{0}\left(\partial_{\phi} \otimes \partial_{t}-\partial_{t} \otimes \partial_{\phi}\right) \\
& +\left(1-2 \lambda_{2}\right)(\gamma+\delta) a_{0}\left(t \partial_{t} \otimes \partial_{t}-\partial_{t} \otimes t \partial_{t}\right)+\mathcal{O}\left(a^{2}\right),
\end{aligned}
$$

where in the lowest order the generators $M_{z t}$ are given as $M_{z t}=z \partial_{t}-t \partial_{z}$. It satisfies Yang-Baxter equation and gives rise to deformed statistics via

$$
\phi(x) \otimes \phi(y)-R \phi(y) \otimes \phi(x)=0 .
$$

\section{CONCLUSIONS}

In this paper we have addressed certain important physics questions that are relevant at the Planck scale. The main physics points discussed here include the nature of particle statistics and the spin-statistics relation at the Planck scale and the deformation of the creationannihilation operator algebra that lies at the core of any quantum field theory at that scale.

The issue of particle statistics and the associated spinstatistics connection is an interesting and open problem in quantum gravity. The arguments presented here indicate that around a noncommutative black hole, the particle statistics is twisted and the oscillators describing a free scalar field satisfy a twisted algebra. The twisted oscillator algebra has a very different structure from its commutative counterpart, although it smoothly reduces to the latter when the deformation parameter is taken to zero.

The deformed oscillator algebra around a noncommutative black hole immediately suggests that there could be a breakdown of the spin-statistics relation in this scenario. Such a violation of the spin-statistics relation in quantum gravity has been previously discussed in the context of quantum geons [31-33]. The violation of the spin-statistics relation observed here is compatible with the fact that the scalar field theory in the background of the $\kappa$-cylinder algebra is nonlocal. In addition, the $\kappa$ Minkowski algebra violates CPT symmetry [48, 49]. It is thus not totally surprising that the usual spin-statistics relation fails to hold in the description of the Planck scale physics presented here. We thus came to the conclusion that the particles in the vicinity of the noncommutative BTZ black hole obey neither Bose, nor Fermi statistics. However, for these particles we managed to write down the dynamical equations which govern their motion in the corresponding background. These equations turn out to be the deformed Klein-Gordon equation (41) describing deformed bosons and the deformed Dirac equation (49) describing deformed fermions.

In this Letter we have worked with a specific realization of the $\kappa$-Minkowski cylinder algebra, which has a smooth limit as the deformation parameter goes to zero. This is an important difference compared to the previous treatment of scalar fields around a noncommutative cylinder in [6, 7]. The twisted flip operator as well as the twisted oscillator algebra

Even though we are dealing with black holes at the Planck scale, the K-G equation (41) appears to be relevant for flat space, which may look like a contradiction. However, let us recall that following the work Doplicher et al 2, 3], the NC setup results from a combined effect of general relativity and quantum uncertainty principle. It is therefore plausible that certain effects of the gravity are already contained in the $\mathrm{NC}$ algebras given by eqns. (3), (7) and (8) of this paper. The KG eqn. (41) is a natural consequence of these algebras which is what we have analyzed here. It would however be interesting to consider the NC metric where the ordinary pointwise products are replaced with the star product that has been obtained here, and to consider the scalar field equation in that background. That requires much further analysis of the underlying noncommutative differential geometry including the behaviour of differential forms under the star products obtained in this paper, which is currently under investigation.

It would also be interesting to study physical processes such as the Hawking radiation around a noncommutative black hole, using the oscillator algebra for the scalar fields presented here, which is a work for the future.

Acknowledgment. This work was supported by the Ministry of Science and Technology of the Republic of Cro- 
atia under contract No. 098-0000000-2865.

[1] S. Majid, Foundations of Quantum Group Theory, (Cambridge University Press, 2000).

[2] S. Doplicher, K. Fredenhagen and J. E. Roberts, Phys.Lett. B 331 (1994) 39.

[3] S. Doplicher, K. Fredenhagen and J. E. Roberts, Commun. Math. Phys. 172 (1995) 187.

[4] B.P. Dolan, Kumar S. Gupta and A. Stern, Class. Quant. Grav. 24 (2007) 1647.

[5] B.P. Dolan, Kumar S. Gupta and A. Stern, J. Phys. Conf. Ser. 174 (2009) 012023.

[6] M. Chaichian, A. Demichev, P. Presnajder and A. Tureanu, Eur. Phys. J. C 20 (2001) 767.

[7] M. Chaichian, A. Demichev, P. Presnajder and A. Tureanu, Phys. Lett. B 515 (2001) 426.

[8] J. Lukierski, A. Nowicki, H. Ruegg and V. N. Tolstoy, Phys. Lett. B 264 (1991) 331.

[9] J. Lukierski, A. Nowicki and H. Ruegg, Phys. Lett. B 293 (1992) 344.

[10] J. Lukierski and H. Ruegg, Phys. Lett. B 329 (1994) 189.

[11] J. Lukierski, H. Ruegg and W. J. Zakrzewski, Ann. Phys. 243 (1995) 90.

[12] P. Schupp and S. Solodukhin, arXiv:0906.2724 [hep-th].

[13] P. Aschieri, C. Blohmann, M. Dimitrijevic, F. Meyer, P. Schupp and J. Wess, Class.Quant.Grav. 22 (2005) 3511.

[14] P. Aschieri, M. Dimitrijevic, F. Meyer and J. Wess, Class. Quant. Grav. 23 (2006) 1883.

[15] A.P. Balachandran, T.R. Govindarajan, K.S. Gupta, S. Kurkcuoglu, Class. Quant. Grav. 23 (2006) 5799.

[16] T. Ohl and A. Schenkel, JHEP 0910 (2009) 052.

[17] G. Amelino-Camelia and S. Majid, Int. J. Mod. Phys. A 15 (2000) 4301.

[18] G. Amelino-Camelia, Int. J. Mod. Phys. D 11 (2002) 35.

[19] J. Kowalski-Glikman, Phys. Lett. A 286 (2001) 391.

[20] J. Kowalski-Glikman and S. Nowak, Phys. Lett. B 539 (2002) 126.

[21] J. Kowalski-Glikman and S. Nowak, Int. J. Mod. Phys. D 12 (2003) 299.

[22] S. Ghosh, Phys. Rev. D 74 (2006) 084019.

[23] S. Ghosh and P. Pal, Phys. Rev. D 75 (2007) 105021.

[24] J.-G. Bu, H.-C. Kim, Y. Lee, C. H. Vac and J. H. Yee, Phys. Lett. B 665 (2008) 95.

[25] H.-C. Kim, Y. Lee, C. Rim and J. H. Yee, Phys. Lett. B 671 (2009) 398.

[26] J.-G. Bu, J. H. Yee, H.-C. Kim, Phys. Lett. B 679 (2009) 486.

[27] G. Amelino-Camelia, L. Freidel, J. Kowalski-Glikman and L. Smolin, arXiv:1101.0931 [hep-th].

[28] G. Amelino-Camelia, N. Loret and G. Rosati, Phys. Lett. B 700 (2011) 150.

[29] G. Gubitosi and F. Mercati, arXiv:1106.5710 [gr-qc].

[30] A. Borowiec, Kumar S. Gupta, S. Meljanac and A. Pachol, Europhys. Lett. 92 (2010) 20006.

[31] J. L. Friedman and R. D. Sorkin, Phys. Rev. Lett. 44 (1980) 1100.

[32] C. Aneziris, A. P. Balachandran, M. Bourdeau, S. Jo, T. R. Ramadas and R. D. Sorkin, Int. J. Mod. Phys. A 4 (1989) 5459.
[33] C. Aneziris, A. P. Balachandran, M. Bourdeau, S. Jo, T. R. Ramadas and R. D. Sorkin, Mod. Phys. Lett. A 4 (1989) 331.

[34] R. Oeckl, Nucl. Phys. B 581 (2000) 559.

[35] M. Chaichian, K. Nishijima and A. Tureanu, Phys. Lett. B 568 (2003) 146.

[36] A. P. Balachandran, G. Mangano, A. Pinzul and S. Vaidya, Int. J. Mod. Phys. A 21 (2006) 3111.

[37] A. P. Balachandran, T. R. Govindarajan, G. Mangano, A. Pinzul, B. A. Qureshi and S. Vaidya, Phys. Rev. D 75 (2007) 045009.

[38] A. P. Balachandran, A. Pinzul, B. A. Qureshi and S. Vaidya, Phys. Rev. D 76 (2007) 105025.

[39] P. Aschieri, F. Lizzi and P. Vitale, Phys. Rev. D 77 (2008) 025037.

[40] M. Arzano and A. Marciano, Phys. Rev. D 76 (2007) 125005.

[41] C. A. S. Young and R. Zegers, Nucl. Phys. B 797 (2008) 537.

[42] M. Daszkiewicz, J. Lukierski and M. Woronowicz, Phys. Rev. D 77 (2008) 105007.

[43] C. A. S. Young and R. Zegers, Nucl. Phys. B 804 (2008) 342.

[44] M. Arzano and D. Benedetti, Int. J. Mod. Phys. A 24 (2009) 4623.

[45] M. Daszkiewicz, J. Lukierski and M. Woronowicz, arXiv:0712.0350

[46] M. Daszkiewicz, J. Lukierski and M. Woronowicz, J. Phys. A 42 (2009) 355201.

[47] J. Lukierski, Rept. Math. Phys. 64 (2009) 299.

[48] T. R. Govindarajan, K. S. Gupta, E. Harikumar, S. Meljanac and D. Meljanac, Phys. Rev. D 77 (2008) 105010.

[49] T. R. Govindarajan, K. S. Gupta, E. Harikumar, S. Meljanac and D. Meljanac, Phys. Rev. D 80 (2009) 025014.

[50] T. R. Govindarajan, Kumar S. Gupta, E. Harikumar and S. Meljanac, J. Phys. Conf. Ser. 306 (2011) 012019.

[51] S. Meljanac and M. Stojić, Eur. Phys. J. C 47 (2006) 531.

[52] S. Meljanac, S. Krešić-Jurić and M. Stojić, Eur. Phys. J. C 51 (2007) 229.

[53] S. Meljanac, A. Samsarov, M. Stojić and K. Gupta, Eur. Phys. J. C 53 (2008) 295.

[54] S. Meljanac and A. Samsarov, Int. J. Mod. Phys. A 26 (2011) 1439.

[55] S. Meljanac, A. Samsarov, J. Trampetic and M. Wohlgenannt, arXiv:1107.2369 [hep-th].

[56] D. Birmingham, Kumar S. Gupta and S. Sen, Phys. Lett. B 505 (2001) 191.

[57] Kumar S. Gupta and S. Sen, Phys. Lett. B 526 (2002) 121.

[58] Sayan K. Chakrabarti, Kumar S. Gupta and S. Sen, Int. J. Mod. Phys. A 23 (2008) 2547.

[59] M. Banados, C. Teitelboim and J. Zanelli, Phys. Rev. Lett. 69 (1992) 1849.

[60] M. Banados, M. Henneaux, C. Teitelboim and J. Zanelli, Phys. Rev. D 48 (1993) 1506.

[61] A. P. Balachandran, T. R. Govindarajan, A. G. Martins and P. Teotonio-Sobrinho, JHEP 0411 (2004) 068. 
[62] S. Meljanac and S. Kresic-Juric, to appear in Int. J. Mod. Phys. A, arXiv:1004.4647 [math-ph].

[63] A. Agostini, F. Lizzi and A. Zampini, Mod. Phys. Lett. A 17 (2002) 2105.

[64] A. Borowiec and A. Pachol, Phys. Rev. D 79 (2009)
045012.

[65] Z. Belhadi, F. Menas, A. Berard, P. Gosselin and H. Mohrbach, arXiv:1106.1302 [hep-th]. 